\title{
Environmental Quality Assessment of Selected Sites in Bolgoda North Lake Using Three Environmental Quality Determining Indices
}

\author{
Fernando M.S.C.* and De Alwis S.M.D.A.U.
}

\author{
Department of Zoology, Faculty of Applied Sciences, University of Sri Jayewardenepura, Nugegoda, \\ Sri Lanka \\ *sheharafdo25@yahoo.com
}

\begin{abstract}
The Index of Biotic Integrity (IBI) based on fish has been identified as a reliable bio monitoring tool that can be used to assess the quality of aquatic ecosystems. This tool was applied to evaluate the habitat quality of three selected sites of the Bolgoda North Lake (6" 40'-49' N: 70" 54'-58' E), a brackish water estuarine system located in the western province of Sri Lanka. In order to compare and contrast the accuracy of IBI based habitat quality assessment, two other common indices, Water Quality Index (WQI) based on selected environmental parameters and Shannon Weiner Diversity Index (SWDI) based on zooplankton and phytoplankton were determined for the three sites.
\end{abstract}

Three selected sites, namely, Thuduwa, Borupana and Attidiya were sampled for fish, selected environmental parameters and zooplankton and phytoplankton over a period of 6 months from February to July 2012. Using 12 metrics that reflected taxonomic composition, habitat composition, tropic composition and fish health, 3 separate fish based Indices of Biotic Integrity were calculated for these three sites.

The environmental quality of the three sites based on the calculated fish based Indices of Biotic Integrity concluded that the condition at Thuduwa site was "good"; at Borupana "poor-very poor" and at Attidiya site "very poor". The calculated WQI values for Thuduwa site qualify it as a site having "good" water quality, Borupana site having "medium" water quality and Attidiya site having "bad" water quality. Results of both indices were generally similar for the three sites. The Shannon Wiener Diversity Index, on the other hand did not show a significant difference between 3 sites, which could be clearly attributable to the high/low evenness values. However, differences shown in the occurrence of indicator species generally agreed with both IBI and WQI. For instance phytoplankton indicators of pollution such as Microcystis sp, Pediastrum sp, Scenedesmus sp, Actinastrum sp were found in high numbers at Attidiya, in moderate numbers at Borupana and only the latter two species in low numbers at Thuduwa.

When all three indices were compared, IBI can be considered as the best index to describe the overall habitat quality, as it reflects the consequences of a long term habitat degradation due to change in environmental parameters at sites shown by changes in fish assemblages whereas WQI reflects the precise level of specific pollutants and SWDI reflects the zooplankton and phytoplankton assemblage at any given time (as they are short lived).

Keywords: Fish based Index of biological integrity, Water Quality Index, Shannon-Weiner diversity index, Bolgoda estuarine system 